\title{
Hypersonic Vehicle Thrust Sensitivity to Angle of Attack and Mach Number
}

\author{
Sean M. Torrez*, James F. Driscoll ${ }^{\dagger}$, Derek J. Dalle* \\ Dept. of Aerospace Engineering, University of Michigan, Ann Arbor MI 48109 \\ Michael A. Bolender ${ }^{\ddagger}$, David B. Doman ${ }^{\S}$ \\ Air Force Research Laboratory, Wright-Patterson AFB, OH 45433
}

Presented at the AIAA Atmospheric Flight Mechanics Conference, Chicago, Illinois, August 2009

\begin{abstract}
A new control-oriented scramjet engine model has been developed, named the MichiganAFRL Scramjet In Vehicle model (MASIV); it is used to compute thrust sensitivity to variation in flight conditions. The model solves conservation equations in 1-D, using several modeling techniques to retain some of the fidelity of higher-order simulations. A number complex physical processes are modeled (including jet mixing and finite rate chemistry) by a combination of ordinary differential equations and algebraic scaling laws. The axial evolutions of the various flow quantities are computed in a short time, relative to computational fluid dynamics solutions. Although there is some loss of accuracy when using Reduced Order Models (ROMs), MASIV computes the overall performance of the flow path with respect to vehicle dynamics (thrust and drag) at an acceptable level for preliminary design and for use as a submodel for control design and evaluation. The model is exercised to predict the sensitivity of the thrust to variations in Mach number and angle of attack, and to compute the operating envelope of the engine.
\end{abstract}

\section{Nomenclature}

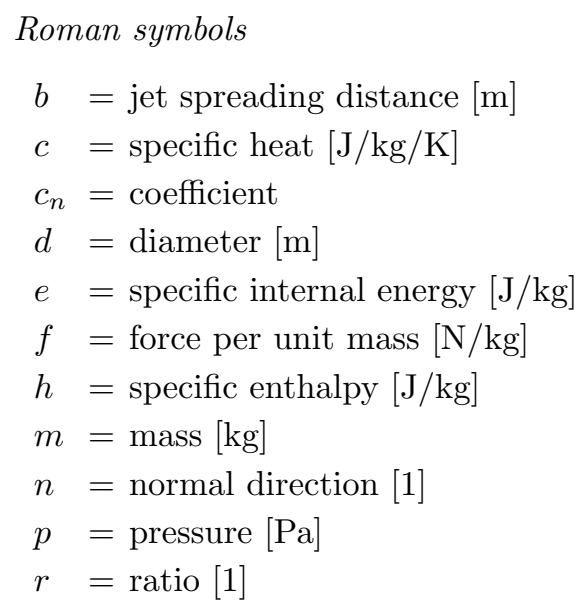

${ }^{*}$ Research Assistant, Student Member, AIAA

$\dagger$ Professor of Aerospace Engineering, Fellow, AIAA

${ }^{\ddagger}$ Aerospace Engineer, AFRL/RBCA, Senior Member AIAA.

$\S$ Senior Aerospace Engineer, AFRL/RBCA, Associate Fellow AIAA. 


$$
\begin{aligned}
u & =\text { axial velocity }[\mathrm{m} / \mathrm{s}] \\
x & =\text { axial coordinate }[\mathrm{m}] \\
y & =\text { transverse vertical coordinate }[\mathrm{m}] \\
z & =\text { transverse horizontal coordinate }[\mathrm{m}] \\
A & =\text { frontal area of duct }\left[\mathrm{m}^{2}\right] \\
C & =\text { concentration }[\mathrm{kmol} / \mathrm{m}] \\
D & =\text { diffusivity }\left[\mathrm{m}^{2} / \mathrm{s}\right] \\
E & =\text { internal energy }[\mathrm{J}] \\
F & =\text { force }[\mathrm{N}] \\
H & =\text { enthalpy }[\mathrm{J}] \\
M & =\text { Mach number }[1] \\
P & =\text { perimeter }[\mathrm{m}] \\
\mathrm{Pr} & =\frac{c_{p} \mu}{k} \text { Prandtl number }[1] \\
Q & =\text { heat }[\mathrm{J}] \\
R & =\text { gas constant }[\mathrm{J} / \mathrm{kg} / \mathrm{K}] \\
S & =\text { area }\left[\mathrm{m}^{2}\right] \\
T & =\text { temperature }[\mathrm{K}] \\
V & =\text { volume }\left[\mathrm{m}^{3}\right] \\
W & =\text { molecular weight }[\mathrm{kg} / \mathrm{kmol}] \\
Y & =\text { mass fraction }[1] \\
\mathcal{R} & =\text { universal gas constant }[8314 \mathrm{~J} / \mathrm{K} / \mathrm{kmol}]
\end{aligned}
$$

Greek symbols

$$
\begin{aligned}
\alpha & =\text { angle of attack }[1] \\
\varepsilon & =\text { momentum injection ratio }[1] \\
\rho & =\text { density }\left[\mathrm{kg} / \mathrm{m}^{3}\right] \\
\sigma & =\text { stress }\left[\mathrm{N} / \mathrm{m}^{2}\right] \\
\chi & =\text { scalar dissipation rate }[1 / \mathrm{s}] \\
\omega & =\text { mass generation }[1] \\
\Phi & =\text { Flux }
\end{aligned}
$$

subscripts

$(\cdot)_{\text {aw }}$ evaluated at adiabatic wall

$(\cdot)_{C L}$ centerline

$(\cdot)_{f} \quad$ friction

$(\cdot)_{h} \quad$ enthalpy conservation

$(\cdot)_{i} \quad$ species index

$(\cdot)_{i n j}$ injected

$(\cdot)_{p} \quad$ constant pressure process

$(\cdot)_{u} \quad$ momentum conservation

$(\cdot)_{v} \quad$ constant volume process

$(\cdot)_{w} \quad$ evaluated at wall

$(\cdot)_{T} \quad$ turbulent

$(\cdot)_{\rho} \quad$ mass conservation

$(\cdot)_{0} \quad$ stagnation quantity

superscripts

ref reference conditions $(T=298 \mathrm{~K}, p=1.00 \mathrm{~atm})$

, fluctuation quantity 


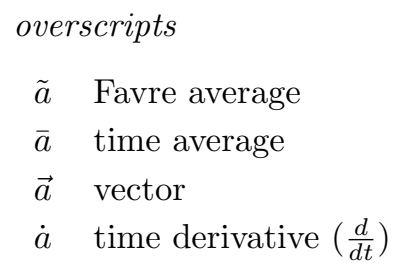

\section{Introduction}

Thrust sensitivity to angle of attack $\left(\frac{\partial F}{\partial \alpha}\right)$ and to flight Mach number $\left(\frac{\partial F}{\partial M_{\infty}}\right)$ are important because they are directly related to the flight dynamics of the hypersonic vehicle. They are especially important because the evolution of flight speed, and flight path angle uniquely determine the position of a 2-dimensional vehicle. Controlling the position of the vehicle in a quasi-steady (trimmed) way is our ultimate goal. These relationships have previously been explored in References [1], [2], [3] and [4].

This study seeks to understand the relationship between thrust and operating parameters for a hypersonic engine flowpath, including inlet, combustor, and nozzle. We computed the variation in thrust for ranges of angle of attack $(\alpha)$ while holding Mach number $(M)$ constant and for ranges of $M$ while holding $\alpha$ constant. Fuel mass flow rate and altitude are held constant for all cases, so the different values of $M$ and $\alpha$ should be considered to be instantaneous perturbations from the design point. This is based on the assumption that variations in fuel mass flow rate and altitude are slow in comparison to variations in $M$ and $\alpha$.

There are two components to the current generation of MASIV: an inlet code which computes wave interactions for arbitrarily shaped bodes, and a combustor code which computes supersonic combustion in arbitrarily shaped ducts with jet fuel injection. The inlet model solves a series of exact Riemann ${ }^{5}$ problems between any two adjacent regions. This gives the classical inviscid solution to any two-wave interaction problem. The model considers shock-shock, shock-expansion and expansion-expansion interactions, modeling expansions as a series of discrete waves.

The combustor code solves a set of differential and algebraic equations in space, ${ }^{6}$ marching axially through the combustion duct. Since combustion in most engines is mixing limited rather than reaction rate limited, jet mixing is computed using the scaling law of Hasselbrink and Mungal, ${ }^{7}$ which is based on flow field similarity. The model considers finite-rate chemistry via the Stationary Laminar Flamelet Model (SLFM), ${ }^{8}$ which considers each point in the flame and maps it to the solution of a corresponding counter-flow flamelet. Because this is a Probability Distribution Function approach, it includes the effects of different strain fields, species diffusion, and momentum diffusion as the duct velocity and fuel jet velocity change.

MASIV computes thrust and drag using a stream tube momentum difference. ${ }^{9}$ It computes the difference between the in-flowing momentum at a given station and the out-flowing momentum at a different station. There are two parts to the flowpath: the inlet, including the forebody, and the combustor, including the internal nozzle. The fixed geometry used in this paper is shown in Figure 2. The component numbering for the flowpath is given in Figure 1.

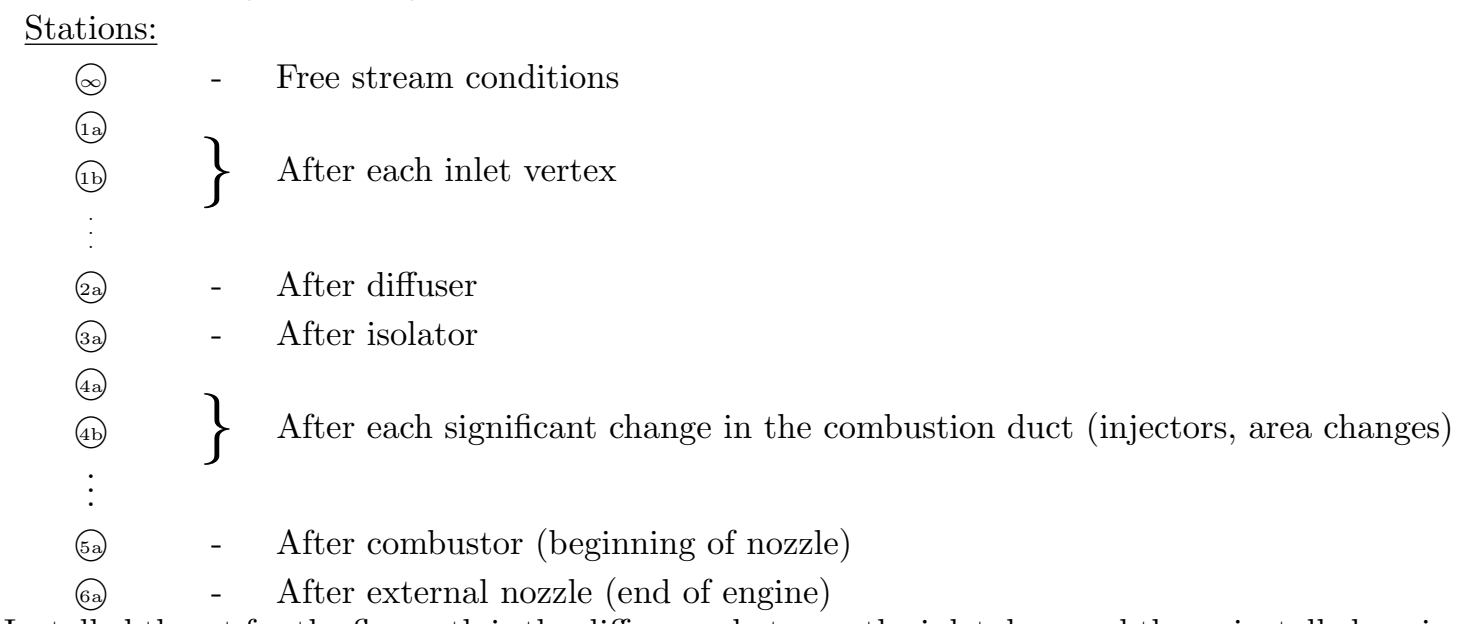

Installed thrust for the flowpath is the difference between the inlet drag and the uninstalled engine thrust. This is similar to the installed thrust of a turbofan or turbojet engine, which usually includes nacelle drag. 


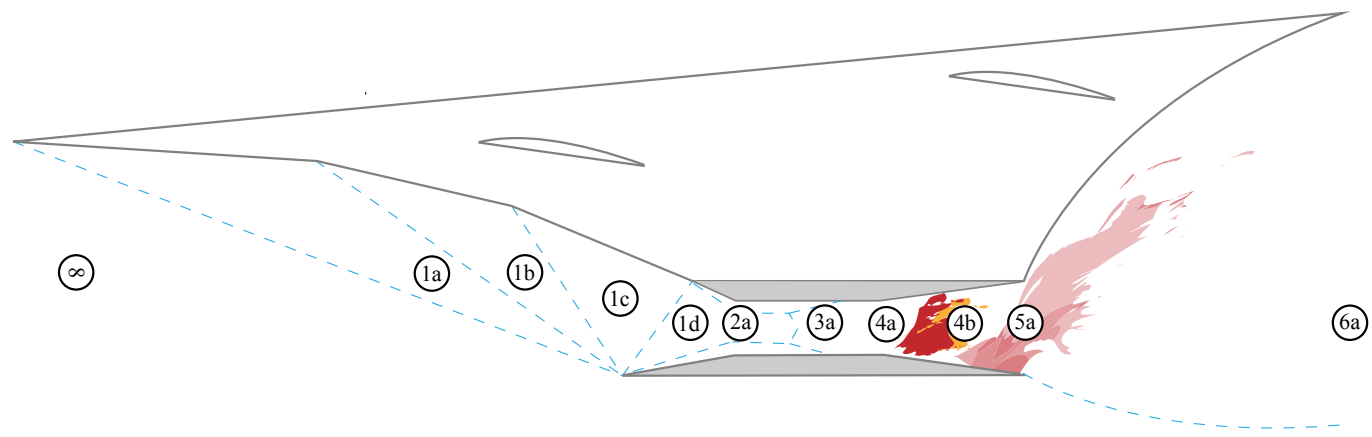

Figure 1. An example geometry. The station numbers are extensible by adding successive letters to each station for a more complex geometry.

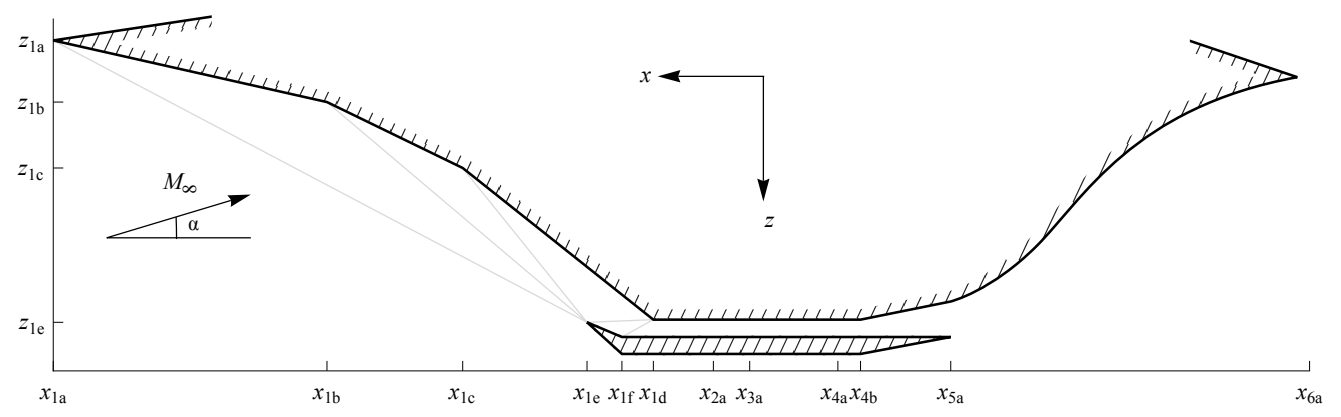

Figure 2. The geometry of the vehicle used in this study is shown. Note that the vertical and horizontal scales are different, though each direction is drawn to scale.

Installed thrust does not include drag from the rest of the vehicle, just drag from air that passes through the engine. The sensitivity of installed thrust to $M$ and $\alpha$ is computed via a finite difference algorithm.

\section{A. Inlet model}

We consider a scramjet inlet design that is approximately 2-dimensional. A satisfactory approach for control application must require a relatively small amount of computational time and still yield a relatively accurate solution to the inlet flow. Instead of solving directly for the flow conditions at each point in the flow (as is done in CFD), this method solves for the positions of the relevant waves, which separate regions in which the flow properties are uniform. The inlet-modeling method solves for the locations of the shock waves and expansions using established two-dimensional supersonic theory. To make this possible in a digital computing environment, expansion fans are approximated as a number of discrete isentropic waves. After determining the locations at which two or more waves intersect, the program solves for the interactions among the waves as a two-dimensional Riemann problem. In many ways this is a generalized and automated version of the method of characteristics.

The input to the program consists of two parts. The first input is the geometry of the inlet, which consists of two polygons: one for the main body of the vehicle and one for the cowl. The second part of the input is the flight conditions, i.e. $M_{\infty}, \alpha$, and altitude. The program begins its analysis slightly upstream of the leading edge and proceeds downstream toward the right-hand $x$-coordinate, $x_{2 \mathrm{a}}$. The program then searches for wave interactions or vertices of the input geometry. As the model is computing the flow solution, there is a current $x$-coordinate. Call this value $x_{\text {cur }}$. This creates a vertical line $x=x_{\text {cur }}$ for which all of the flow upstream has been computed, and all of the flow downstream remains to be solved. There will be several waves that intersect this vertical line at vertical coordinates of $z_{1}, z_{2}, \ldots$, each wave having a corresponding propagation angle, $\sigma_{1}, \sigma_{2}, \ldots$.

The first $x$-coordinate of interest is the first leading edge of the vehicle. At this point two shock waves are introduced: one above the leading edge and one below. Also, two additional trajectories are tracked, which correspond to the upper and lower boundaries of the vehicle body. All of these trajectories start with 
the same $z$-coordinate (that of the leading edge) but different values of $\sigma_{j}$. Because the trajectories all have different propagation angles, they will become farther apart as the program progresses downstream from the leading edge. With the set of waves and surfaces at a given $x$-coordinate given, a list of possible intersection points can be determined. Suppose that the trajectories at the current $x$-coordinate are listed by ascending $z$-coordinates. Then two trajectories with coordinates $z_{j}$ and $z_{j+1}$ have a downstream intersection point of

$$
x_{\mathrm{next}, j}=x_{\mathrm{cur}}+\frac{z_{j+1}-z_{j}}{\tan \sigma_{j+1}-\tan \sigma_{j}}
$$

provided that $\sigma_{j}<\sigma_{j+1}$. Then the $x$-coordinate of the next interaction point, $x_{\text {next }}$ is the minimum of all the $x_{k, j}$ and all of the vertices of the input polygons that are downstream of $x_{\text {cur }}$.

Once an interaction point is found, the nature of the interaction is determined, and the local flow problem is solved. If the interaction is a wave (shock or discrete expansion wave) interacting with either the vehicle body or the cowl, the downstream conditions are determined by either a shock or an expansion. If the interaction consists of two waves interacting with each other, the solution is determined by a Riemann problem. In this situation both the flow above the interaction point and the flow below the interaction point pass through an additional wave, and the strengths of the new waves are such that their post-wave pressures are equal. Before moving to the next interaction point, the list of waves and their propagation angles at the current $x$-coordinate are updated so that the coordinates of the next interaction point can be determined accurately.

Once the solution has reached the beginning of the isolator at $x_{2 \mathrm{a}}$, it is useful to define the spatially averaged gas properties, which can be input into a one-dimensional isolator or combustor model. The proposed method to determine spatially-averaged properties is to require that the total fluxes of mass, momentum, and stagnation enthalpy are constant through the $x=x_{2 \mathrm{a}}$ plane. This method is chosen so that the averaging does not violate conservation of mass or energy and introduces no drag. The combination of the inlet model and the averaging method will provide a flow solution in less than ten seconds for all of the flight conditions considered in this report.

\section{Combustion Modeling}

The MASIV code marches the flow conservation equations from the beginning of the combustor (station 3a) to the end of the internal nozzle (station 5a). All flow states are allowed to vary in the downstream axial direction, defined $-\hat{x}$. We allow derivatives with respect to the axial coordinate only. Some quantities, such as jet spreading and mixing, vary in the transverse directions, but they may only vary algebraically, such that their evolutions do not depend on the information propogating downstream.

\section{A. Duct Solution}

We begin with the conservation equations, along with the equation of state.

Table 1. Conservation equations used in MASIV.

\begin{tabular}{lc}
\hline \hline Equation Name & Equation \\
\hline Equation of State & $p=\rho \frac{\mathcal{R}}{W} T$ \\
Cons. of Mass & $\frac{\partial}{\partial t} \iiint_{V} \rho d V+\iint_{S} \rho \vec{u} \cdot \vec{n} d S=0$ \\
Conservation of Species & $\frac{\partial}{\partial t} \iiint_{V} Y_{i} \rho d V+\iint_{S} Y_{i} \rho \vec{u} \cdot \vec{n} d S=\dot{\omega}$ \\
Cons. of Momentum & $\frac{d}{d t} \iiint_{V} \rho \vec{u} d V=-\iint_{S}(\rho \vec{u}) \vec{u} \cdot \vec{n} d S+\iiint_{V} \rho \vec{f} d V+\iint_{S} \overline{\bar{\sigma}} \vec{n} d S$ \\
Cons. of Energy & $\frac{d}{d t} \iint_{V}\left(e+\frac{\vec{u} \cdot \vec{u}}{2}\right) d V+\iint_{S} \rho\left(e+\frac{\vec{u} \cdot \vec{u}}{2}\right) \vec{u} \cdot \vec{n} d S+\iint_{S} p \vec{u} \cdot n d S=\iiint_{V} \rho \vec{f} \cdot \vec{u} d V+\dot{Q}+\dot{W}$ \\
\hline \hline
\end{tabular}

We take the derivatives of these equations with respect to $x$ to obtain a set of ODEs, which are presented in Table 2. These equations form the basis of the model, since their simultaneous solution provides all the information needed to calculate the evolutions of the state variables $(p, \rho, T)$ and the heat release through the duct. 
Since the specific heat capacity, $c_{p}$ of each species varies with $T$, which varies through the duct, using $c_{p}$ to calculate enthalpy offers no advantage, so we avoid formulating the problem in this way. The JANAF and GRIMech tables are readily available (CHEMKIN ${ }^{10}$ is one convenient source), so we simply use the enthalpy $(h)$ curve fits, and circumvent a messy formulation for $c_{p}$. If the specific heat is desired, it can be computed a posteriori.

Similarly, the sound speed ( $a$ ) for a reacting flow is not defined $a=\sqrt{\gamma R T}$, but instead must be defined as $a=\left(\frac{\partial p}{\partial \rho}\right)_{s=\text { const. }}$. We avoid this difficulty by formulation the problem in terms of velocity rather than Mach number. This is what Heiser and Pratt ${ }^{11}$ call enthalpy-kinetic energy $(H-K)$ space. It is more convenient than the typical temperature-Mach number $(T-M)$ space, giving a simpler set of equations. If desired, $a$ and $M$ can be computed post-solution as well as $c_{p}$.

Table 2. Differential equations used in MASIV.

\begin{tabular}{ll}
\hline \hline ODE Name & Equation \\
\hline Equation of State & $\frac{1}{p} \frac{d p}{d x}=\frac{1}{T} \frac{d T}{d x}+\frac{1}{\rho} \frac{d \rho}{d x}-\frac{1}{W} \frac{d W}{d x}$ \\
Cons. of Mass & $\frac{1}{\rho} \frac{d \rho}{d x}=\frac{1}{\dot{m}} \frac{d \dot{m}}{d x}-\frac{1}{u} \frac{d u}{d x}-\frac{1}{A} \frac{d A}{d x}$ \\
Cons. of Species & $\frac{d Y_{i}}{d x}=\frac{\dot{\omega}}{\rho u}+\frac{1}{\dot{m}} \frac{d \dot{m}_{i}}{d x}-\frac{Y_{i}}{\dot{m}} \frac{d \dot{m}}{d x}$ \\
Cons. of Momentum & $\frac{1}{u} \frac{d u}{d x}=-\frac{1}{\rho u^{2}} \frac{d p}{d x}-\frac{C_{f}}{2 A} \frac{d S_{w}}{d x}-\frac{(1-\varepsilon)}{\dot{m}} \frac{d \dot{m}}{d x}$ \\
Cons. of Energy & $\frac{1}{h_{0}} \frac{d T}{d x} \sum_{i} c_{p, i} Y_{i}=-\frac{u}{h_{0}} \frac{d u}{d x}-\frac{1}{\dot{m}} \frac{d \dot{m}}{d x}+\frac{1}{h_{0} \dot{m}} \frac{\rho u C_{f}\left(h_{a w}-h_{w}\right)}{2 P r^{2} / 3} \frac{d S_{w}}{d x} \cdots$ \\
& $\quad+\frac{1}{h_{0} \dot{m}} \frac{\rho u^{3} C_{f}}{2} \frac{d S_{w}}{d x}-\frac{1}{h_{0}} \sum_{i} h_{i} \frac{d Y_{i}}{d x}+\frac{1}{h_{0} \dot{m}} \sum_{i} h_{i} \frac{d \dot{m}_{i}}{d x}$ \\
\hline \hline
\end{tabular}

Clearly, there are several other quantities indicated in Table 2 which are required to solve the system, but which are not part of the solution. These are presented in Table 3.

\section{B. Chemistry}

We compute the reaction rate $\left(\widetilde{\dot{\omega}_{i}}\right)$ for each species using the SLFM before solving the set of ODEs. ${ }^{12}$ The SLFM uses a counter-flow flamelet solution with an assumed PDF model for each flow parameter: mean mixture fraction $(\tilde{f})$, mean mixture fraction variance $\left(\tilde{f}^{\prime}\right)$ and mean scalar dissipation rate $(\widetilde{\chi})$. This means that for any given point in the flow, $\widetilde{\dot{\omega}}_{i}$ can be determined as a function of $\tilde{f}, \tilde{f}^{\prime}$ and $\tilde{\chi}$. The SLFM equation follows.

$$
-\chi_{s t} \frac{\partial \psi}{\partial f}=\frac{\dot{\mathbf{m}}}{\rho}
$$

Here, $\boldsymbol{\psi}$ and $\dot{\boldsymbol{m}}$ represent the vectors

$$
\boldsymbol{\psi}=\left[\begin{array}{c}
Y_{1} \\
\vdots \\
Y_{n} \\
h
\end{array}\right] \quad \dot{\mathbf{m}}=\left[\begin{array}{c}
\rho \dot{\boldsymbol{\omega}}_{1} \\
\vdots \\
\rho \dot{\boldsymbol{\omega}}_{n} \\
-\dot{q}_{R}
\end{array}\right]
$$

This results in a flamelet lookup table of reaction rates for each species, $\dot{\mathbf{m}}\left(f, \chi_{s t}\right)$. The solution of the flamelet equations has been discussed sufficiently in the literature (see [8]), so discussion will be omitted here.

Once a flamelet solution has been found, we require mean values of each variable in order to use the mixing model discussed in Section C. This is accomplished using a probability density function approach which assumes a certain PDF and variance for each quantity, then integrates the product over the parameter space. 
Table 3. Additional equations used in MASIV.

\begin{tabular}{ccl}
\hline \hline Source & Variable & Equation \\
\hline From Solution & $=\int \frac{d p}{d x} d x$ \\
$\rho$ & $=\int \frac{d \rho}{d x} d x$ \\
$Y_{i}$ & $=\int \frac{d Y_{i}}{d x} d x$ \\
$u$ & $=\int \frac{d u}{d x} d x$ \\
$T$ & $=\int \frac{d T}{d x} d x$ \\
\hline Given & $=A(x)$ \\
$\frac{d A}{d x}$ & $=\frac{d A}{d x}(x)$ \\
$\frac{d \dot{m}_{i}}{d x}$ & $=\frac{d \dot{m}_{i}}{d x}(x)$ \\
$\dot{\omega}$ & $=\dot{\omega}(x)$ \\
$W_{i}$ & $=c o n s t .^{\dagger}$ \\
$C_{f}$ & $=c o n s t$. \\
$\dot{m}$ & $=\rho u A$ \\
$\frac{d W}{d x}$ & $=-W^{2} \sum_{i}\left(\frac{1}{W_{i}} \frac{d Y_{i}}{d x}\right)$ \\
$\frac{d S_{w}}{d x}$ & $=\sqrt{16 A+\left(\frac{d A}{d x}\right)^{2}}$ \\
$\frac{d \dot{m}}{d x}$ & $=\sum_{i} \frac{d \dot{m}_{i}}{d x}$ \\
$h_{i}$ & $=h_{i}(T)^{\dagger}$ \\
$h$ & $=\sum_{i} h_{i}$ \\
$h_{0}$ & $=h+\frac{u^{2}}{2}$ \\
$h_{w}$ & $=h\left(T_{w}\right)$ \\
$h_{a w}$ & $=h+\sqrt{\operatorname{Pr} \frac{u^{2}}{2}}$ \\
\hline \hline & &
\end{tabular}

${ }^{\dagger}$ from CHEMKIN

For example, to compute the mean of the reaction source terms, which we desire as reaction rate input into the duct solution code, the following equation applies.

$$
\widetilde{\mathbf{m}}\left(\tilde{f}, \widetilde{f^{\prime \prime 2}}, \bar{\chi}_{s t}\right)=\int_{0}^{\infty} \int_{0}^{1} \dot{\mathbf{m}}\left(f, \chi_{s t}\right) \widetilde{P}(f) P\left(\chi_{s t}\right) d f d \chi_{s t}
$$

where $\widetilde{P}(f)$ is the beta PDF, $\beta\left(f, \tilde{f}, \widetilde{f^{\prime \prime 2}}\right)$ and $P\left(\chi_{s t}\right)$ is a log-normal PDF. Finally, a mapping from $\tilde{\chi}$ to $\bar{\chi}_{s t}$ is required, which can be accomplished using another PDF

$$
\tilde{\chi}=\bar{\chi}_{s t} \int_{0}^{1} F(f) \beta\left(f, \tilde{f}, \widetilde{f^{\prime \prime 2}}\right) d f
$$

These functions are tabulated for a given chemistry to improve solution speed. In principle, it is possible to pre-tabulate chemistry for any given configuration. In application, it is best to use a limited number of chemistry files in order to reduce the amount of memory and disk space required.

\section{Assumed Mixing Model}

The jet centerline penetration and spreading can be computed for each point using the scaling relationship measured in [13]. This relationship is valid for momentum ratio $r_{u} \gg 1$. Experimental results set the values of the constants in Table 4, which allow MASIV to generate a predicted three-dimensional distribution of fuel based on injection and crossflow parameters. 
First, the momentum ratio is defined as

$$
r_{u}=\left[\frac{\rho_{i n j}}{\rho}\left(\frac{u_{i n j}}{u}\right)^{2}\right]^{1 / 2}
$$

which appears in the scaling relationship

$$
\frac{y_{C L}}{d_{i n j}}=c_{1}\left(\frac{x_{C L}}{d_{i n j}}\right)^{c_{2}} r_{u}^{2 / 3}
$$

to trace the centerline path of the fuel jet.

The normalized concentration of injected fuel is given by another scaling relationship:

$$
\frac{C_{C L}}{C_{i n j}}=c_{3}\left(\frac{\rho_{i n j}}{\rho}\right)^{1 / 3}\left(\frac{u_{i n j}}{u}\right)^{-1 / 3}\left(\frac{x_{C L}}{d_{i n j}}\right)^{-2 / 3}
$$

The mean mixture fraction is assumed to be 1 in the injected gas stream (pure fuel) and 0 in the cross flow (pure oxidizer):

$$
\begin{aligned}
m & =\frac{W_{i n j}}{W} \\
\tilde{f}_{C L} & =\frac{C_{C L} m}{1+(m-1) C_{C L}} \\
\tilde{f}(s, n) & =\tilde{f}_{C L} \exp \left(\frac{-n^{2}}{2 b}\right)
\end{aligned}
$$

The mixture fraction at a given point is determined by the centerline mixture fraction corresponding to that point, and by the jet spreading distance, which is a function of distance from the injector along jet centerline.

$$
\begin{aligned}
n^{2} & =\left(x-x_{C L}\right)^{2}+\left(y-y_{C L}\right)^{2}+z^{2} \\
\frac{b}{d_{i n j}} & =c_{4} r_{u}^{2 / 3}\left(\frac{x_{C L}}{d_{i n j}}\right)^{1 / 3}
\end{aligned}
$$

Thus, by computing the shortest perpendicular distance from a given point to the jet centerline, the mixture fraction can be computed. The constants $c_{1}-c_{5}$ are experimentally determined. Their values in MASIV are given in Table 4.

Table 4. Experimental constants for jet mixing model.

\begin{tabular}{c|c|c}
\hline \hline Constant & Experimental Range & MASIV value \\
\hline$c_{1}$ & 1.2 to $2.6^{13}$ & 2.1 \\
$c_{2}$ & 0.28 to $0.34^{13}$ & $\frac{1}{3}$ \\
$c_{3}$ & $0.85-0.95^{14}$ & 0.9 \\
$c_{4}$ & $0.35^{7}$ & 0.35 \\
$c_{5}$ & 0.14 & 0.14 \\
\hline \hline
\end{tabular}

The mean mixture fraction variance is computed by another scaling argument. Measurements indicate that mixture fraction variance is essentially a function of rate of change of mean mixture fraction, so that

$$
\begin{aligned}
& \tilde{f}^{\prime}=c_{5} \frac{\partial f}{\partial r} \\
& \tilde{f}^{\prime}=c_{5} \frac{r}{b} f
\end{aligned}
$$

Finally, we determine the local scalar dissipation rate, $\tilde{\chi}$ using the formula: 


$$
\begin{aligned}
& \chi=2 D|\nabla f|^{2} \\
& \tilde{\chi}=2 D_{T}|\nabla \tilde{f}|^{2}
\end{aligned}
$$

where $D$ is the scalar diffusion coefficient and $D_{T}$ is the turbulent scalar diffusion coefficient. We can estimate $D_{T}$ using $^{15}$

$$
\begin{aligned}
\frac{u_{0} d}{\nu_{T}} & \approx 65 \\
\mathrm{Sc}_{T} & =\frac{\nu_{T}}{D_{T}} \\
& \approx 0.55
\end{aligned}
$$

\section{Solution Procedure}

To solve for combustion in the duct, we first define a geometry $\left(A, \frac{d A}{d x}\right)$ and a fuel injection profile $\left(\frac{d \dot{m}}{d x}\right)$ as functions of axial distance, $x$. For a given fuel injection location, we know the mass flow rate of fuel that enters the duct. Based on the fuel mass flow rate, the jet scaling law gives us a 3-dimensional flow field in which

$$
\begin{aligned}
\tilde{f} & =\tilde{f}(x, y, z) \\
\tilde{f}^{\prime} & =\widetilde{f}^{\prime}(x, y, z) \\
\widetilde{\chi} & =\widetilde{\chi}(x, y, z) \\
\widetilde{\dot{\omega}}_{i} & =\widetilde{\dot{\omega}}_{i}\left(\tilde{f}, \widetilde{f}^{\prime}, \widetilde{\chi}\right) \\
\widetilde{\dot{\omega}}_{i} & =\widetilde{\dot{\omega}}_{i}(x, y, z)
\end{aligned}
$$

We then integrate $\dot{\omega}(x, y, z)$ to determine the 1 -dimensional rate of reaction of each species:

$$
\widetilde{\dot{\omega}}_{i}(x)=\iint \widetilde{\dot{\omega}}_{i}(x, y, z) d y d z
$$

With $\dot{\omega}(x)$ we now have enough information to solve the equations in Table 2. A stiff solver is required, due to the rapid reaction rates. We used MATLAB's ode23tb.

\section{Installed Thrust Sensitivity}

We define the thrust sensitivity to be $\frac{d F}{d Q}$, where $Q$ is some flight condition (e.g., $M, \alpha, h$, etc.) or some control input ( $\phi$, control surface deflection, etc.). In this paper, we consider installed thrust sensitivity to $M$ and $\alpha$. Ideally, $\frac{d F}{d M}$ and $\frac{d F}{d \alpha}$ will be small so that the vehicle will be easier to control.

\section{A. Factors Affecting Thrust}

The thrust is computed using a streamtube momentum difference. We select the streamtube such that it contains all the air that goes through the engine and nothing more. It begins at the nose of the vehicle (since no information can propogate forward in the supersonic flow) and ends at the end of the nozzle, which we assume isentropically expands the flow to ambient pressure. Using these assumptions, it is not necessary to consider pressure contributions to thrust. The thrust equation then simplifies to

$$
\begin{aligned}
F & =\dot{m}_{\text {out }} u_{\text {out }}-\dot{m}_{\text {in }} u_{\text {in }} \\
& =\rho_{6 a} u_{6 a}^{2} A_{6 a}-\rho_{\infty} u_{\infty}^{2} \cos ^{2}(\alpha) A_{\text {capture }}
\end{aligned}
$$


We normalize the (installed) thrust by the captured momentum flux, $\Phi_{u}=\rho_{\infty} u_{\infty}^{2} \cos ^{2}(\alpha) A_{\text {capture }}$

$$
\begin{aligned}
\frac{F_{\text {inst }}}{\rho_{\infty} u_{\infty}^{2} \cos ^{2}(\alpha) A_{\text {capture }}} & =\frac{\rho_{6 a} u_{6 a}^{2} A_{6 a}}{\rho_{\infty} u_{\infty}^{2} \cos ^{2}(\alpha) A_{\text {capture }}}-1 \\
\frac{F_{\text {inst }}}{\Phi_{u}} & =\frac{\dot{m}_{6 a}}{\dot{m}_{1 a}} \frac{u_{6 a}}{u_{1 a}}-1 ;
\end{aligned}
$$

Similarly, the normalized inlet drag and normalized uninstalled thrust may be computed

$$
\begin{aligned}
\frac{F_{\text {uninst }}}{\Phi_{u}} & =\frac{\dot{m}_{6 a}}{\dot{m}_{1 a}} \frac{u_{6 a}}{u_{1 a}}-\frac{\dot{m}_{3 a}}{\dot{m}_{1 a}} \frac{u_{3 a}}{u_{1 a}} \\
\frac{D_{\text {inlet }}}{\Phi_{u}} & =1-\frac{\dot{m}_{2 a}}{\dot{m}_{1 a}} \frac{u_{2 a}}{u_{1 a}}
\end{aligned}
$$

It is clear that velocity lost due to shocks in the inlet will decrease the installed thrust. Uninstalled thrust and inlet drag are broken out separately to illustrate how inlet losses affect the installed thrust of the engine. Since the flowpath is inviscid, losses are only possible through pressure drag and wave drag.

Usually, thrust is considered to be directly proportional to mass flow through the engine. In the case of a scramjet engine, however, the relationship is less simple. Figure 3 graphically shows the wave pattern in the inlet. Note that increasing angle of attack increases the area of the captured streamtube, increasing mass flow through the engine. Similarly, increasing Mach number increases mass flow through the engine, since the velocity is larger, causing larger flux through the capture area. In the hypersonic case however, the shock pattern in the inlet must be considered.

Pressure losses to due an excessive number of shocks reduce the stagnation pressure of the flow so significantly that thrust is seriously affected, due to both the pressure loss itself and also to reduced combustion efficiency. Considering even small perturbations from the design condition (for example $M=7.9$ or $M=8.1$ and $\alpha=-1^{\circ}$ or $\alpha=1^{\circ}$ ) we see that the shock pattern changes visibly. If the change is such that more shocks are "swallowed" by the inlet, which causes them to interact with each other as well as the interior walls of the engine, losses are substantial.

Therefore, under conditions with Mach number smaller than $M_{\text {design }}$ or angle of attack larger than $\alpha_{\text {design }}$, more shocks are present in the inlet and losses are large. For example, consult Figures $3 \mathrm{~b}$ and 3o.

\section{B. Sensitivities}

Since the thrust values are only computed at discrete points, jumps in value between adjacent points cause the derivative computed by finite difference to be large. To avoid this, the derivative is computed based on the smoothed functions (using the loess method). This causes some loss of information, but the derivatives shown present the features of the sensitivity much more clearly than the jagged, unsmoothed versions.

The sensitivities of Installed Thrust to $M$ and $\alpha$ are presented in Figures 4 to 9 . Note that the engine is most sensitive to variations near the design point. This is undesirable, since we want the vehicle to be stable near its trim condition. There appears to be a nearly linear relationship between $M$ and $F$, with $F$ decreasing as $M$ increases, except for the sudden decrease just before the design point and the sudden increase right at the design point. The relationship between $\alpha$ and $F$ has its peak right around the design point, except for the sudden decrease just after the design point.

Both of these sensitivities reveal sudden changes in installed thrust, even for small parameter variations, which present a serious control challenge.

\section{Operating Envelope}

The operating envelope of the flowpath (that is, the boundary on which the flowpath produces zero installed thrust) is presented in Figure 10. Note that the envelope is not bounded on the left or right because the installed thrust of the engine never reaches zero for high or low Mach numbers. With vehicle drag in place, this would change. The operating envelope allows the control system to select appropriate bounds on control input and selection of realistic flight trajectories. 


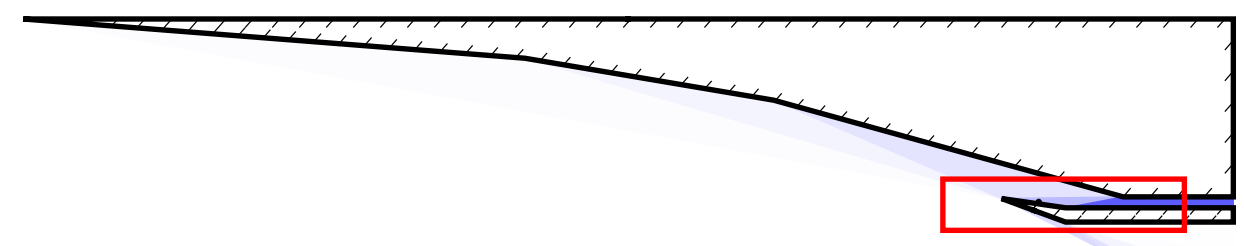

a) The inlet at full scale with $\alpha=0^{\circ}, M=8$

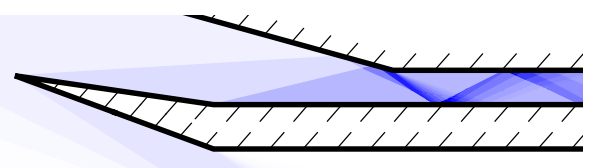

b) $\alpha=0^{\circ}, M=6$

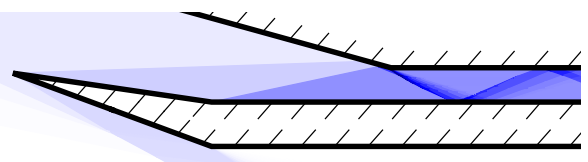

d) $\alpha=0^{\circ}, M=7$

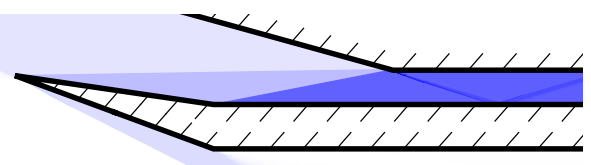

f) $\alpha=0^{\circ}, M=7.9$

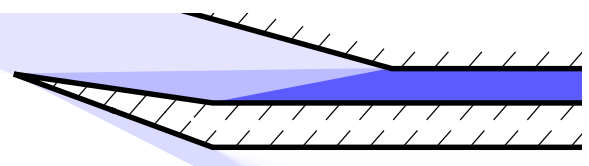

h) $\alpha=0^{\circ}, M=8$

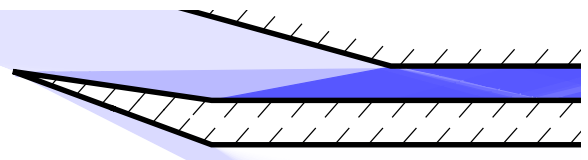

j) $\alpha=0^{\circ}, M=8.1$

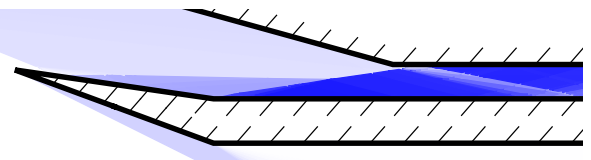

1) $\alpha=0^{\circ}, M=9$

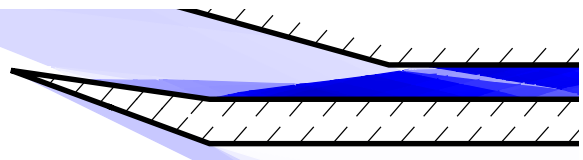

n) $\alpha=0^{\circ}, M=10$

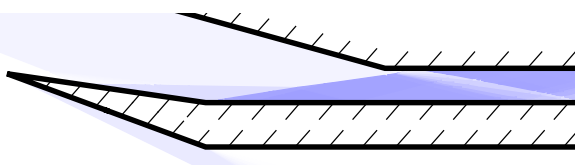

c) $\alpha=-5^{\circ}, M=8$

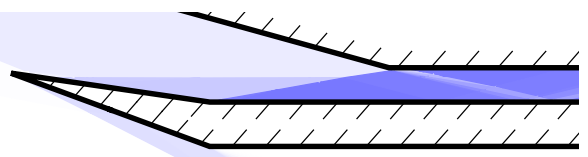

e) $\alpha=-2^{\circ}, M=8$

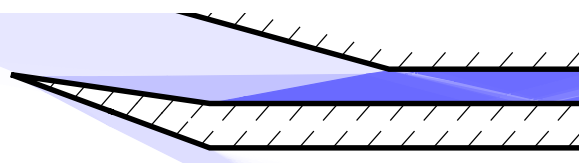

g) $\alpha=-1^{\circ}, M=8$

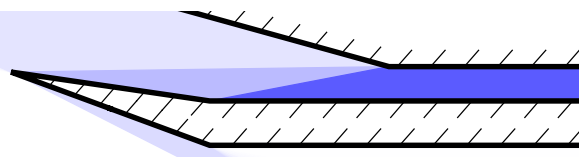

i) $\alpha=0^{\circ}, M=8$

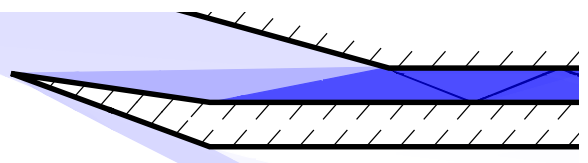

k) $\alpha=1^{\circ}, M=8$

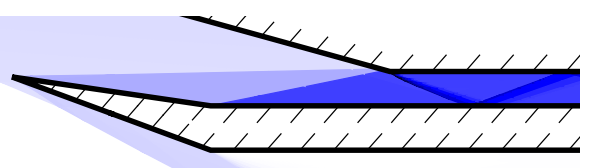

m) $\alpha=2^{\circ}, M=8$

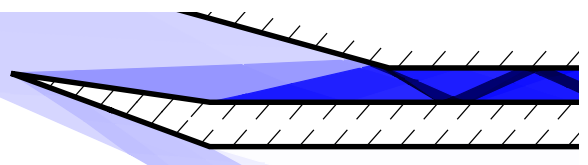

o) $\alpha=5^{\circ}, M=8$

Figure 3. The discretized inviscid flow through the inlet for a series of $\alpha$ and a series of $M_{\infty}$. The top image shows the entire inlet at the design condition. The smaller images show the indicated portion of the inlet for a variety of Mach numbers and angles of attack. Darker shades of blue represent regions of higher pressure; white represents freestream pressure and black represents $p / p_{\infty}=200$. 


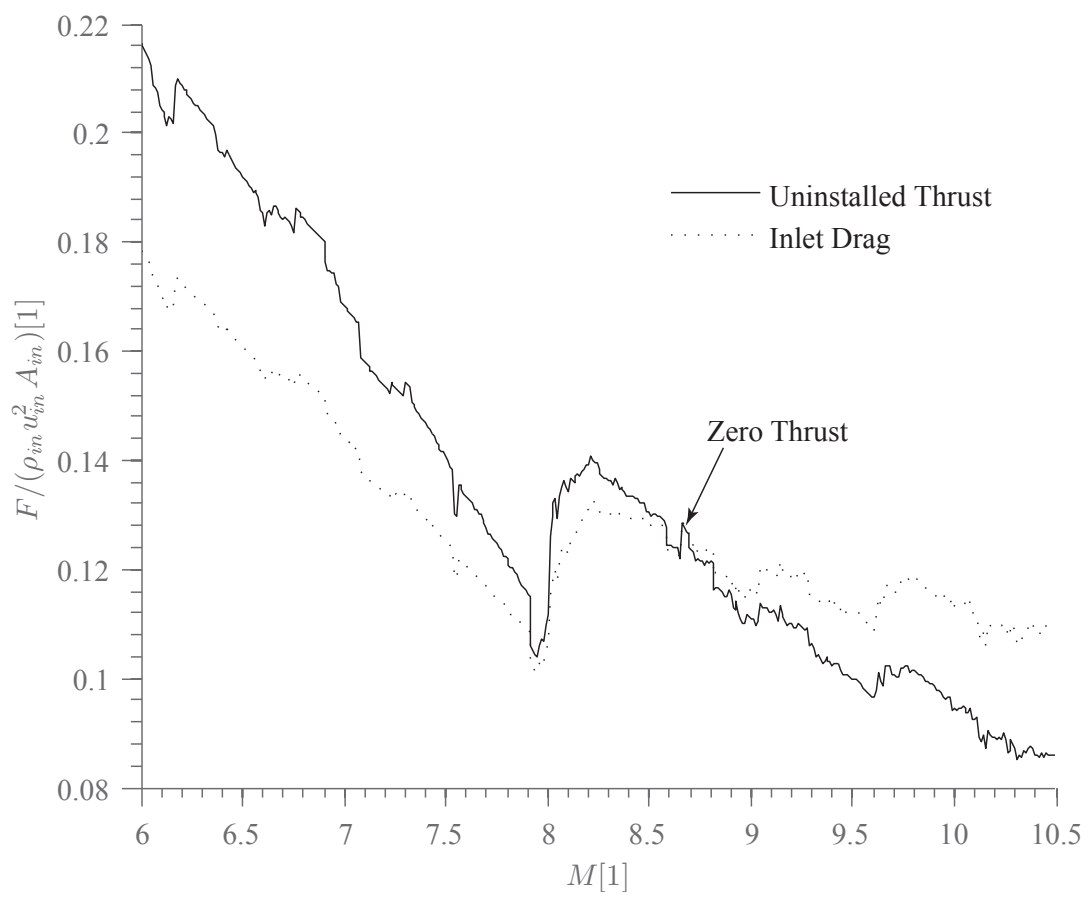

Figure 4. Uninstalled thrust and inlet drag vs. Mach number, $M=[6,10.5], \alpha=0^{\circ}$

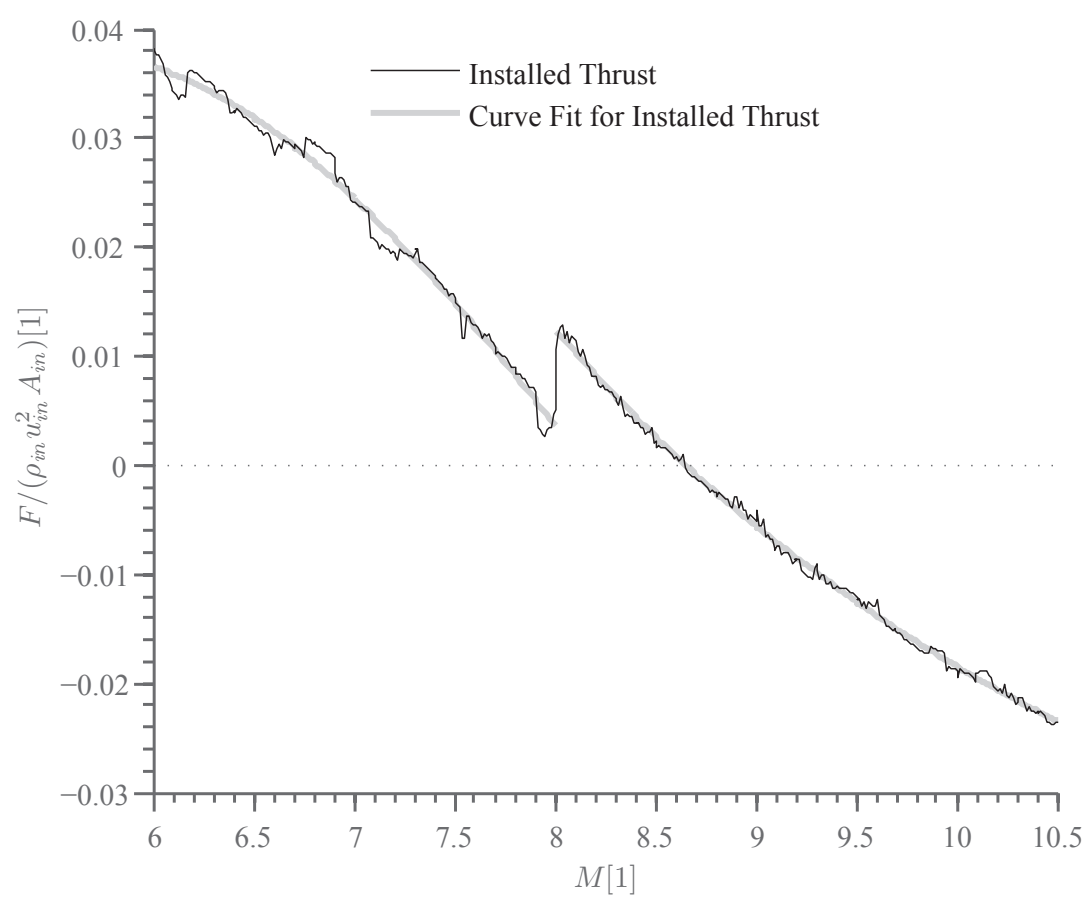

Figure 5. Installed thrust vs. Mach number, $M=[6,10.5], \alpha=0^{\circ}$ 


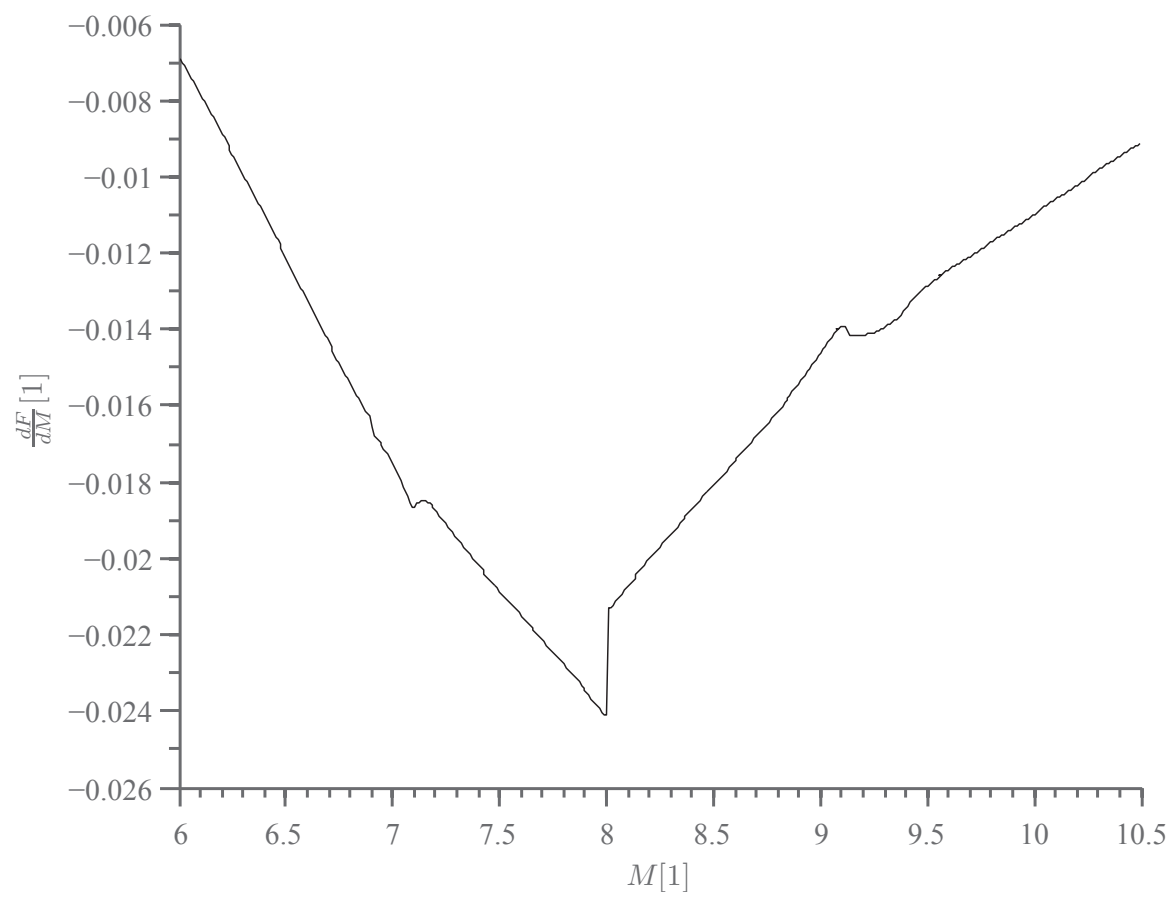

Figure 6. Sensitivity of installed thrust to Mach Number vs. Mach number, $M=[6,10.5], \alpha=0^{\circ}$

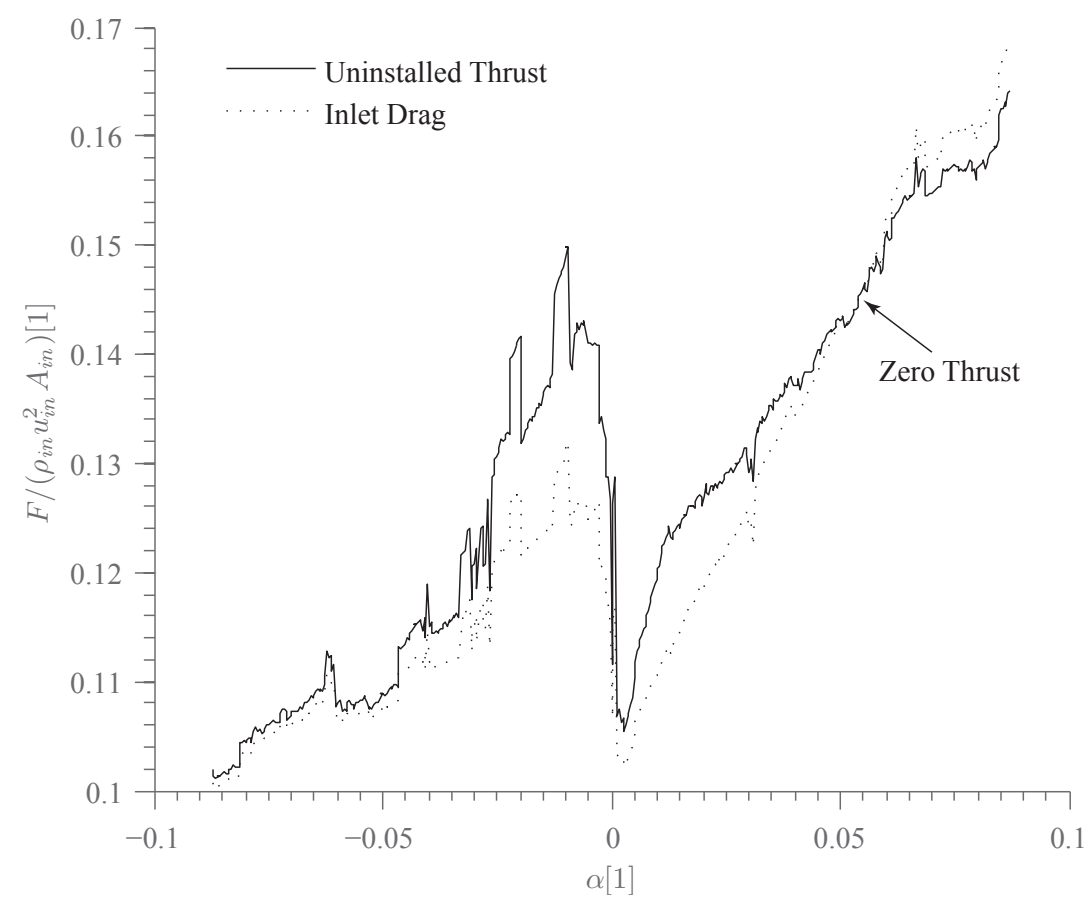

Figure 7. Uninstalled thrust and inlet drag vs. angle of attack, $M=8, \alpha=\left[-5^{\circ}, 3.8^{\circ}\right]$ 


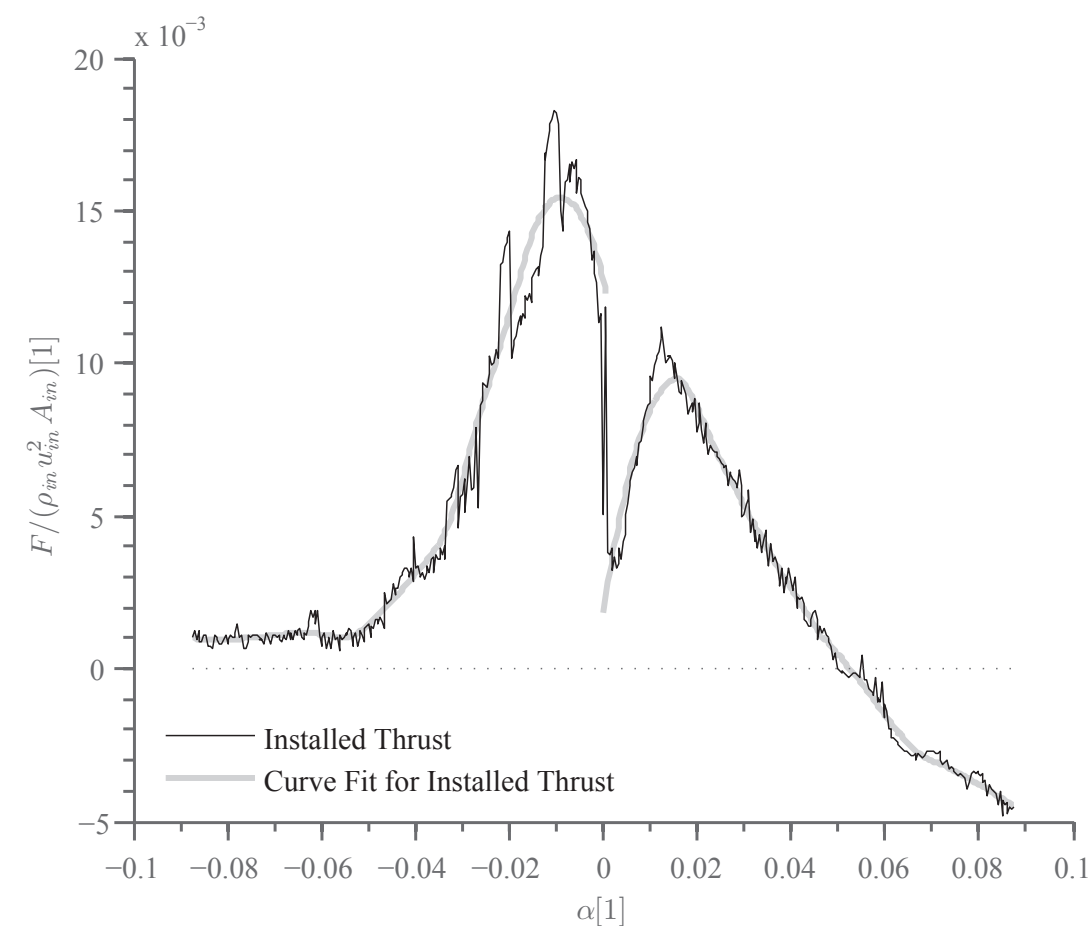

Figure 8. Installed thrust vs. angle of attack, $M=8, \alpha=\left[-5^{\circ}, 3.8^{\circ}\right]$

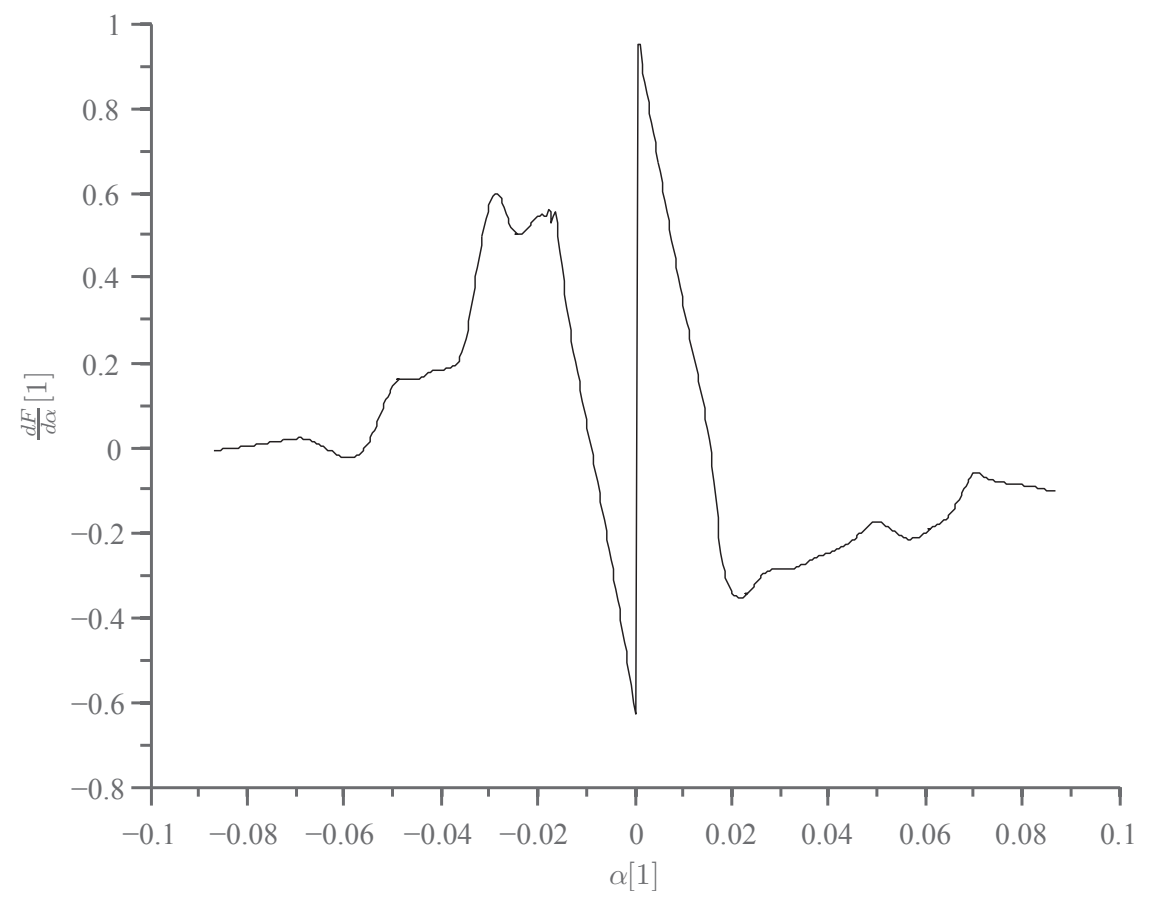

Figure 9. Sensitivity of installed thrust to angle of attack vs. angle of attack, $M=8, \alpha=\left[-5^{\circ}, 3.8^{\circ}\right]$ 


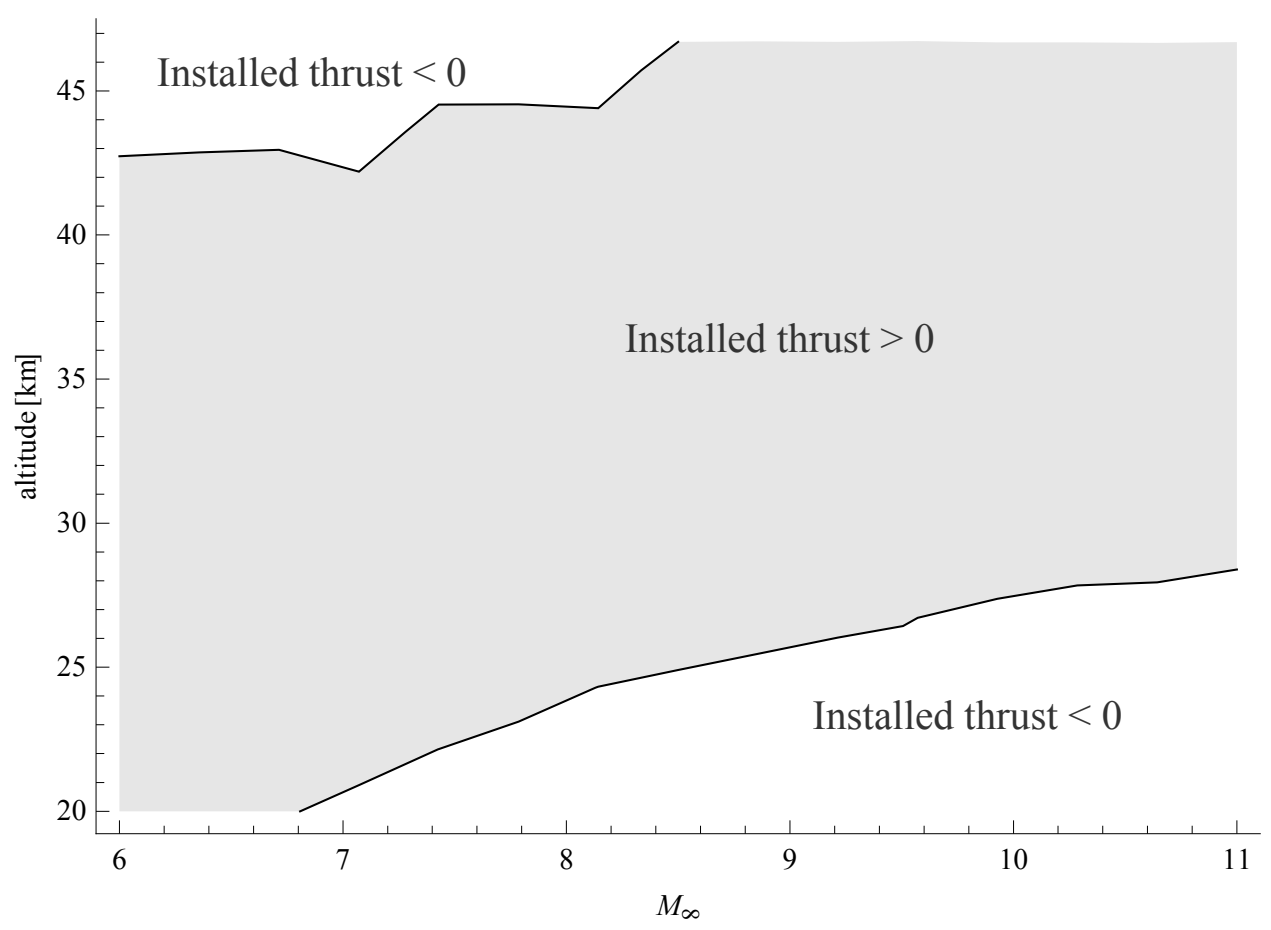

Figure 10. Flight envelope of the flowpath.

\section{Conclusions}

The main conclusion is that the thrust sensitivity to $\alpha$ and $M$ when wave interactions in the inlet and realistic combustion are considered curves are nearly the opposite of simple models. When $\alpha$ or $M$ is increased, mass flow through the engine increases. However, in the hypersonic vehicle, this increase in mass flow can be offset by the effect of pressure losses in the inlet, which cause a great deal of drag. Inlets for hypersonic vehicle engines must be carefully designed to reduce this effect.

Complex chemistry can also have a direct effect on thrust. The compression efficiency of the inlet varies with $M$ and $\alpha$, changing the combustor pressure and temperature over a wide range and reducing combustion efficiency when the pressure and temperature are low. Heat release and area profiles in scram-mode engines must be designed to operate over wide pressure and temperature ranges.

\section{Acknowledgements}

This research was supported by AFRL grant FA 8650-07-2-3744 for the MACCCS (Michigan AFRL Collaborative Center for Control Science) and by NASA grant NNX08AB32A, administered by Donald Soloway and Peter Ouzts, technical monitors.

\section{References}

\footnotetext{
${ }^{1}$ Torrez, S. M., D. J. F. D. D. J. and Micka, D. J., "Scramjet Engine Model MASIV: Role of Mixing, Chemistry and Wave Interactions," AIAA-2009-4939.

${ }^{2}$ Torrez, S. M.; Scholten, N. M. D. D. J. F. B. M. D. D. and Oppenheimer, M. W., "A Scramjet Engine Model Including Effects of Precombustion Shocks and Dissociation," AIAA 2008-4619.

${ }^{3}$ Torrez, S. M. Driscoll, J. F. B. M. A. O. M. W. and Doman, D., "Effects of Improved Propulsion Modelling on the Flight Dynamics of Hypersonic Vehicle," 2008.

${ }^{4}$ Bolender, M. A. and Doman, D. B., "Nonlinear Longitudinal Dynamical Model of an Air-Breathing Hypersonic Vehicle," Journal of Spacecraft and Rockets, Vol. 44, No. 2, March/April 2007, pp. 374-387.

${ }^{5}$ Anderson, J. D., Modern Compressible Flow, McGraw Hill, 3rd ed., 2003.

${ }^{6}$ O'Brien, T. F.; Starkey, R. P. and Lewis, M. J., "Quasi-One-Dimensional High-Speed Engine Model with Finite-Rate Chemistry," Journal of Propulsion and Power, Vol. Vol. 17, No. 6, 2001, pp. pp. 1366-1374.
} 
${ }^{7}$ Hasselbrink, E. F. and Mungal, M. G., "Transverse jets and jet flames. Part 2. Velocity and OH field imaging," Journal of Fluid Mechanics, Vol. 443, 2001, pp. 27-68.

${ }^{8}$ Peters, N., "Laminar Diffusion Flamelet Models in Non-Premixed Turbulent Combustion," Progress in Energy Combustion Science, Vol. 10, 1984, pp. 319-339.

${ }^{9}$ Kerrebrock, J. L., Aircraft Engines and Gas Turbines, MIT Press, 1992.

${ }^{10}$ R. J. Kee, F. M. Rupley, J. A. M. M. E. C. J. F. G. E. M. H. K. M. A. E. L. G. D.-L. M. D. S. J. W. G. H. E. R. S. L. R. E. M. L. R. P. W. C. R. M. C. W. E. S. P. G. C. W. C. L. M. O. A. W. G. H. C. P. C. S. F. M. P. H. P. D. Y. D. J. Y. D. W. H. M. V. P. and Puduppakkam, K. V., CHEMKIN Release 4.1, Reaction Design, San Diego, CA, 2006.

${ }^{11}$ Heiser, W. and D., P., Hypersonic Airbreathing Propulsion, American Institute of Aeronautics and Astronautics, 370 L'Enfant Promenade, SW,Washington D.C., 20024-2518, 1994, pp. 109-143.

${ }^{12}$ Pitsch, H., "FlameMaster v3.1: A C++ computer program for 0D combustion and 1D laminar flame calculations." 1998.

${ }^{13}$ Hasselbrink, E. F. and Mungal, M. G., "Transverse Jets and Jet Flames: Part 1. Scaling Laws for Strong Transverse Jets," Journal of Fluid Mechanics, Vol. Vol. 443, 2001, pp. pp. 1-25.

${ }^{14}$ Smith, S. H. and Mungal, M. G., "Mixing, structure and scaling of the jet in crossflow," Journal of Fluid Mechanics, Vol. 357, 1998, pp. 83-122.

${ }^{15}$ Peters, N., "Die Struktur turbulenter Freistrahl-Diffusionsflammen," Chemie Ingenieur Technik, Vol. 55 No. 10, 1983, pp. $743-751$. 